\title{
Integrated Yoga Practice in Cardiac Rehabilitation Program: A Randomized Control Trial
}

\author{
K.N. Srihari Sharma, MPT,1,2 Subramanya Pailoor, PhD (Yoga), ${ }^{1}$ Nidhi Ram Choudhary, PhD (Yoga), \\ Prabhavathi Bhat, MD, DM, DNB (Cardiology), ${ }^{3}$ and Smeeta Shrestha, $\mathrm{PhD}^{4}$
}

\begin{abstract}
Background: Coronary artery disease (CAD) is a detrimental noncommunicable disease, which is increasing due to sedentary lifestyle and urbanization in the young population. It is further elevated with risk factors such as stress, anxiety, depression, an increase in triglycerides, dyslipidemia, hyperglycemia, hypertension, and so on, which manifests as atherosclerotic disease. Yoga-based lifestyle intervention is a noninvasive effective treatment method to control and prevent cardiac risk factors in CAD patients. Yoga has been used in India as a therapeutic method to manage hypertension and other chronic disorders and is fast gaining popularity as an effective means for the alleviation of stress, improvement of fitness, and enhancement of well-being. This study aimed to determine the feasibility of introducing the integrated approach of yoga therapy (IAYT) in a cardiac rehabilitation center in India and understand its usefulness in improving the cardiac function and managing the cardiac risk factors in acute myocardial infarction patients with left ventricular dysfunction.

Methods and Design: Cardiac patients were randomized to a yoga-practicing group $(n=33)$ and a control group $(n=33)$. The yoga-practicing group was instructed to attend three supervised IAYT classes 3 days per week for 12 weeks at the hospital yoga center. The control group received standard care that included pharmacologic treatment and the instructions of the cardiologist. The outcome measures were assessed at baseline $(\mathrm{T} 1=0)$ and completion $(\mathrm{T} 2=3$ months). The primary outcome measure was the left ventricular ejection fraction (LVEF).

Results: There was no statistically significant difference in LVEF $(U=420.500, p$ value $=0.218)$ between the two groups. However, the yoga-practicing group showed significant reduction in depression (Cardiac Depression Scale $[\mathrm{CDS}], U=71, p$ value $=0.0$ ), anxiety (Hamilton Anxiety Rating Scale [HAM-A], $U=128$, $p$ value $=0.0)$, and a significant increase in quality of life $(\mathrm{QOL})$ scores (Duke Activity Status Index [DASI], $U=146, p$ value $=0.0$; and metabolic equivalents $($ METs), $U=136, p$ value $=0.0)$ at 3 months compared to control. Overall, the CAD patients practicing yoga showed a favorable profile compared to control individuals on CDS, HAM-A, DASI, and MET outcomes. Control and yoga practicing groups did not differ significantly in the lipid levels.

Conclusion: This study indicated that the integration of yoga practice in a cardiac rehabilitation program is feasible and has no added benefit in improving the cardiac function. However, the addition of yoga to cardiac rehabilitation may be beneficial in reducing depression and anxiety and improving QOL in patients.
\end{abstract}

Keywords: IAYT, yoga, rehabilitation, myocardial infarction, CAD

\footnotetext{
${ }^{1}$ Division of Yoga and Life Sciences, Swami Vivekananda Yoga University (SVYASA), Bangalore, India.

${ }^{2}$ College of Physiotherapy, School of Health Sciences, Dayananda Sagar University, Bangalore, India.

${ }^{3}$ Department of Cardiology, Sri Jayadeva Institute of Cardiovascular Sciences and Research, Bangalore, India.

${ }^{4}$ School of Basic and Applied Sciences, Dayananda Sagar University, Bangalore, India.
} 


\section{Introduction}

$\mathbf{C}$ ORONARY ARTERY DISEASE (CAD) is associated with impaired cardiovascular functioning and is the leading cause of morbidity and mortality globally. ${ }^{1}$ It is a chronic noncommunicable disease with a prevalence of $11 \%$ in urban and $5 \%$ in rural settings in India. ${ }^{2}$ Urbanization, sedentary lifestyle, and other associated risk factors such as dyslipidemia, hyperglycemia, high triglycerides, and hypertension further elevate the chronic disease. These factors, along with obesity, increase endothelial injury further developing to atherosclerosis, which causes cardiac arrest. ${ }^{3,4}$ Cardiovascular events such as myocardial infarction (MI) and stroke constitute primary manifestations of atherosclerosis, ${ }^{2}$ followed by anxiety, depression, reduced activity, and poor quality of life (QOL), ${ }^{5}$ as secondary symptoms.

The left ventricular ejection fraction (LVEF) measures the left ventricular systolic function. The LVEF is a powerful predictor of cardiac mortality, and the accurate measurement of LVEF is very important for managing patients with cardiovascular disease. LVEF values $>50 \%$ are normal, while those $<30 \%$ are severely abnormal. ${ }^{6,7}$ Lower percentages of LVEF are associated with unfavorable longterm prognosis in CAD patients. ${ }^{8}$

Lipids are known to play a key role in the development of CAD. ${ }^{3}$ The pathophysiology of atherogenesis and oxidative stress is directly linked to the oxidation of low-density lipoprotein (LDL) particles. ${ }^{9}$ Elevated risk of CAD has been linked with an increased ratio of total cholesterol to highdensity lipoprotein (HDL) cholesterol. ${ }^{10}$ Raised plasma triglycerides are associated with type 2 diabetes, which in turn is considered to be a strong risk factor for CAD. ${ }^{11}$

High cholesterol levels are responsible for around 56\% of CAD worldwide. ${ }^{12}$ This increased total cholesterol and a diminished fraction of HDL among the adult population, with co-occurrence of hypertension, smoking, diabetes, and obesity, are the major determinants of clinical manifestation of atherosclerotic disease, which further elevates CAD. ${ }^{13-16}$

Following acute MI, patients are adversely affected by the secondary manifestations of atherosclerosis, namely, anxiety and depression, resulting in a profound impact on their CAD prognosis. ${ }^{17-19}$ Psychologic risk factors are $\sim 10 \%$ more prevalent among the CAD population with $15 \%-20 \%$ cardiac patients exhibiting depressive symptoms. ${ }^{20}$ The first 2 years of follow-up after MI are considered crucial because of the high risk of developing anxiety and depression, which further leads to recurrent MI. ${ }^{20,21}$ The American heart association recommends routine screening for depression in patients with CAD in various health care settings, and professionals qualified in the diagnosis and management of depression evaluate these patients. ${ }^{22}$

Cardiac patients, especially the elderly, experience difficulty in activities of daily living, including self-care, which is crucial for a satisfactory QOL. ${ }^{23}$ In CAD patients, angina, fatigue, dyspnea, and depression are important symptoms that limit QOL. Previous studies of CAD patients have demonstrated a direct relationship between individual functional status and QOL. ${ }^{24}$

Cardiac rehabilitation programs have demonstrated a $40 \%-70 \%$ reduction in the prevalence of anxiety, depression, hostility, and up to $70 \%$ reduction in the risk of mortality. ${ }^{8,18}$ Clinical guidelines with respect to comprehensive cardiac rehabilitation clearly suggest psychologic interventions, ${ }^{8}$ but an absence of standard recommendations for the same is an impeding factor at the patient level. Furthermore, patient referral and participation in such rehabilitation programs are sparse. Managing psychologic distress is important to support cardiac patients in the restoration of their QOL and functional capacity. Serious efforts to explore practical solutions in this area of behavioral cardiology are in progress. ${ }^{20,25,26}$

Assessment of health-related QOL as a clinical endpoint in chronically ill patients has gained importance over the past few decades. ${ }^{24}$ Particular attention toward interventions that enhance QOL through the promotion of self-care is necessary for the reduction of symptoms of both physical and psychologic origin. ${ }^{23,27,28}$

The epidemiologic transition toward urbanization tends to raise the plasma cholesterol levels in the population largely due to a shift in the consumption of dietary fats and reduction of physical activity. ${ }^{12}$ The use of the lipid profile as a screening procedure for CAD patients may be beneficial. ${ }^{14,15,29,30}$ Numerous measures to lower the serum cholesterol levels, including lifestyle modifications, exercises, and physical activities, should be considered complementary to lipid-lowering drugs to keep MI and CAD at bay. ${ }^{15,31}$

Yoga, a mind-body therapy, would be a promising therapeutic and health promotional strategy in the management of risk factors associated with CAD. ${ }^{32}$ Yoga enhances psychologic well-being by reducing psychologic morbidity, modifying reactivity to stressors, adapting stress-related coping mechanisms, minimizing the symptoms of anxiety and depression, reducing tension, anger, and fatigue, improving sleep quality, and enhancing the QOL. ${ }^{32}$ Figure 1 illustrates how yoga helps to establish collateral pathways toward health and well-being by breaking the vicious circle of progressive emotion, the cognitive, structural, and psychologic disturbance that would lead to the development and aggravation of CAD risk factors. ${ }^{27}$

Raghuram et al. investigated yoga-based cardiac rehabilitation programs for patients who had undergone coronary artery bypass grafting (CABG). Patient follow-up demonstrated improvements in LVEF, body mass index, serum glucose, and lipids and a decrease in stress, anxiety, and depression. ${ }^{8}$ Another study, which practiced 12 weeks of Hatha Yoga intervention in Chinese adults, suggested yoga to be a good alternative to modify cardiac risk factors owing to its low-to-moderate intensity and a high adherence rate. ${ }^{33}$

The article covers a single-center study designed to evaluate the feasibility and efficacy of integrated approach of yoga therapy (IAYT) based cardiac rehabilitation program in a center in India, adjunct to conventional pharmacologic management in improving the cardiac function, minimizing the symptoms of anxiety and depression, and moderately normalizing the lipid profile in acute MI patients with left ventricular dysfunction.

\section{Materials and Methods}

\section{Study design}

The study was conducted between 2015 and 2017 at Sri Jayadeva Institute of Cardiovascular Sciences and Research, Bangalore, Karnataka, India. 


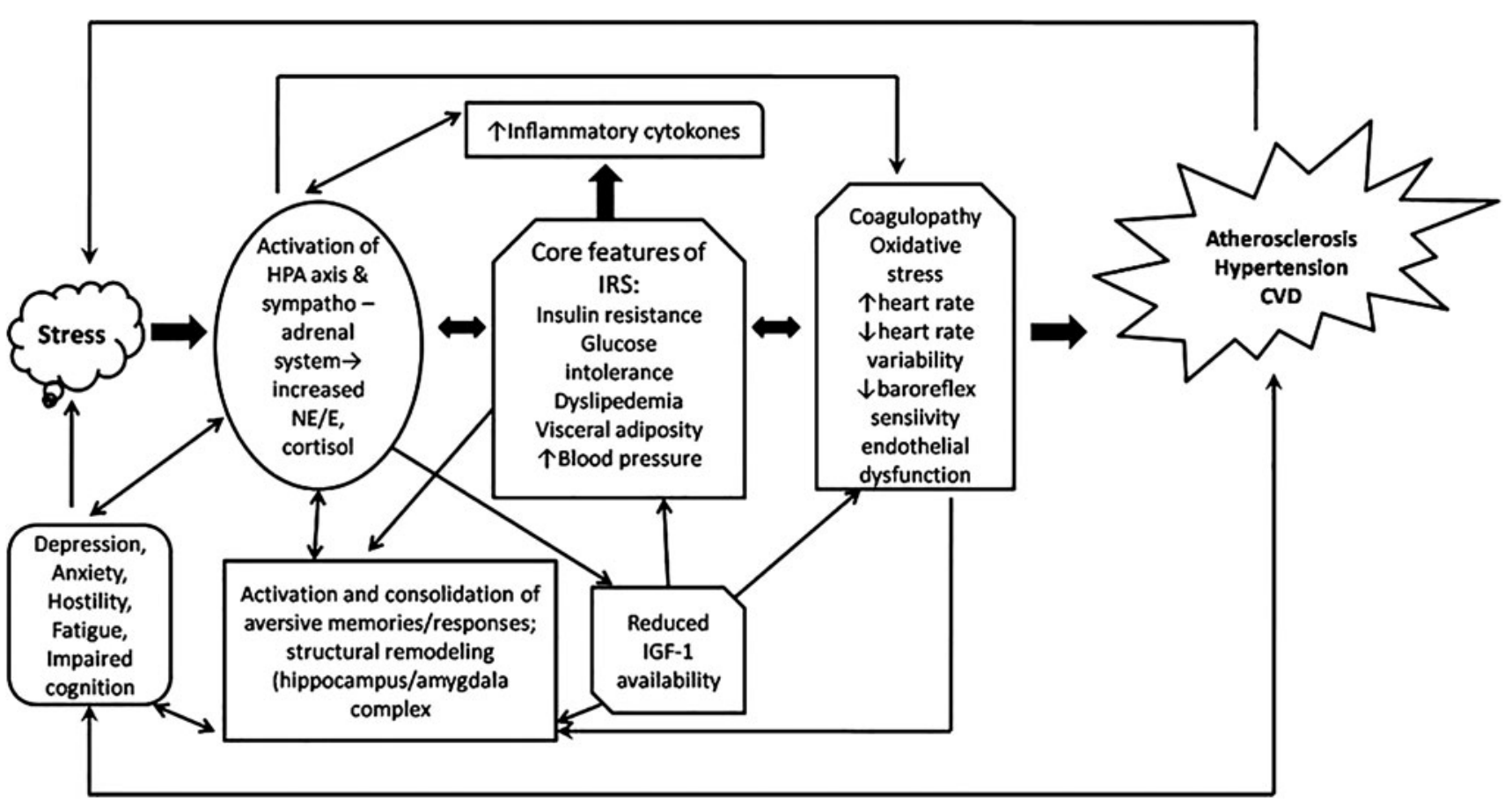

FIG. 1. Schematic illustration of the effect of stress in the human body. CAD, cardiovascular disease; E, epinephrine; HPA, hypothalamic pituitary adrenal; IGF, insulin-like growth factor; IRS, insulin resistance syndrome; NE, norepinephrine.

\section{Participants and recruitment}

Eligibility criteria. The study was open to adults of both genders (age 30-65 years) who were able to consent for themselves (presumed from completion of the consent form). Patients were included if they had (1) recent MI on conservative medical management-without involving any revascularization procedures like Percutaneous Coronary Intervention or CABG (10 days-2 months post-MI), (2) left ventricular dysfunction (New York Heart Association [NYHA] classes I and II), and (3) LVEF between 30\% and $50 \%$. Patients were excluded if (1) they had LVEF $<30 \%$, (2) class III and IV heart failure, (3) unstable cardiac symptoms like angina, (4) recurrent ischemia, (5) concurrent pulmonary disease like chronic obstructive pulmonary disease, (6) uncontrolled arrhythmia, (7) severe musculoskeletal disease like osteoarthritis restraining the patient to perform yoga, (8) hypertension (systolic blood pressure of $>160 \mathrm{mmHg}$ or diastolic blood pressure of $>100 \mathrm{mmHg}$ ), (9) valvular heart disease, and (10) patients with hematologic, renal, or hepatic dysfunction (Fig. 2).

Sample size. G*Power version $3.1 .3^{34}$ was used to calculate the sample size based on the results of a previous study. ${ }^{35}$ A priori computation of the required sample size with a probability error alpha $=0.05$, for a power of 0.8 , yielded a result of 48 as the total sample size with 24 in each group. Anticipating dropout, the authors recruited an additional $20 \%$ so that their sample constituted a total of 66 patients.

Randomization. Randomization was stratified by two groups - control and yoga. The participants were randomly assigned to either of the groups at a 1:1 ratio. The sequential sampling method was adopted, in which an interim analysis was done after reaching the determined sample size a-priori (16 in each group) with the power of the sample size of 80 , and based on the results, additional participants were recruited to acquire the adequate power of the study. The allocation was determined based on minimization using the software MinimPy ${ }^{36}$ with the following randomization parameters: Biased coin minimization probability method, Marginal balance distance measure, and 0.7 Base probability. The baseline characteristics of the study population are given in Table 1. The cardiac sonographer, laboratory staff, and the subjects were blinded to the group. The analyst performed the randomization using MinimPy 0.3, and the group status was written in a concealed slip of paper and this was directed to the cardiac patient through yoga therapists.

Intervention. The participants were randomly assigned to two groups, namely, Group 1-yoga and Group 2-control. Compilation and an expert validation of a yoga module for the current randomized control trial (RCT) were performed (Supplementary Table S1). ${ }^{37}$ The yoga group received $1 \mathrm{~h}$ supervised yoga module comprising of Asanas (physical postures), Pranayama (breathing techniques), and relaxation techniques thrice a week for 12 weeks along with the standard pharmacologic therapy prescribed for the condition. Certified yoga therapists with a minimum qualification of Masters of Science (M.Sc.) in yoga and a minimum experience of 3 years in the field of yoga conducted the sessions in the hospital yoga center under the supervision of senior yoga therapists/cardiologists. The patients were encouraged to practice the same $1 \mathrm{~h}$ yoga, as per the module, at home during the other days of the week, for which the participants were provided with handouts and a digital versatile disc 


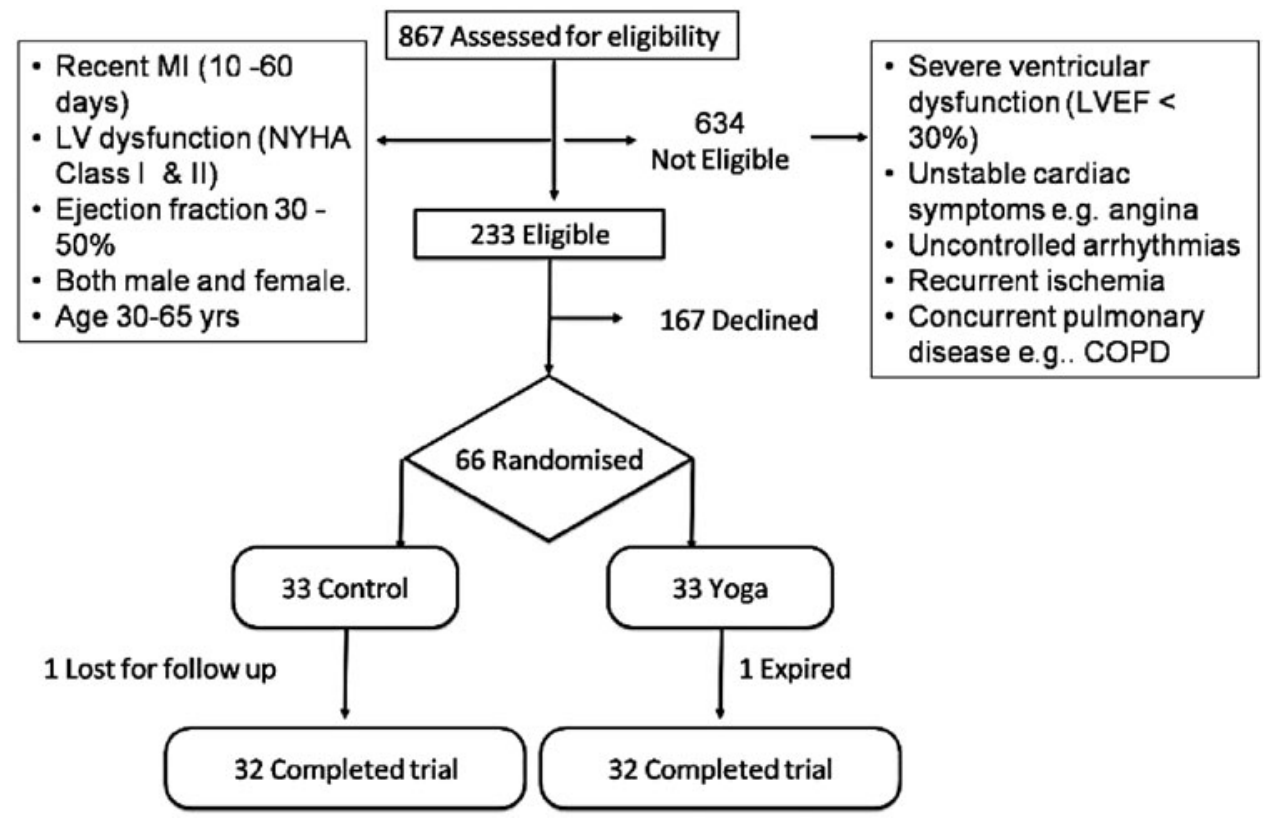

FIG. 2. Flow chart illustrating study outline. COPD, chronic obstructive pulmonary disease; LVEF, left ventricular ejection fraction; MI, myocardial infarction; NYHA, New York Heart Association.

consisting of explanation and demonstration of the practices for their convenience. Participants were instructed to mark the days of their home practice in a diary.

Control group. The control group received standard care that included pharmacologic treatment and the instructions of the cardiologist.

Outcome measures. Demographic data, including age, sex, education, employment status, marital status, socioeconomic status, and ethnicity, were recorded. Cardiac measures: LVEF was assessed to evaluate the cardiac function using standard trans thoracic echocardiography, which is the imaging of choice recommended for the measurement of LVEF. ${ }^{8,38}$ The assessment of the QOL of the participants was performed using the Duke Activity Status Index (DASI). ${ }^{39}$ DASI is a valid and reliable self-administered functional capacity tool that measures the performance of a set of 12 common activities of daily living under four major activity domains. Metabolic equivalents (METs) were derived from DASI scores. ${ }^{40,41}$ Biochemical measures: The total plasma triglycerides (Tg), cholesterol, LDL, and HDL were assessed using the Shotgun technique. ${ }^{3}$ Psychosocial measures: Anxiety and depression were assessed using the Hamilton Anxiety Rating Scale (HAM-A) and the Cardiac Depression Scale (CDS), respectively. HAM-A is a 14-item self-administered anxiety rating scale widely used in clinical and research settings. Each item is scored on a scale of 0 (not present) to 4 (severe), with a total score range of 0-56. ${ }^{42}$ Depression was measured using a 26-item CDS questionnaire, each item of which may be graded on a 7-point Likert scale ranging from "strongly disagree" to "strongly agree". ${ }^{43,44}$ Data on all outcome measures were collected at the baseline and on completion of 12 weeks.

Table 1. Baseline Characteristics of the Study Subjects

\begin{tabular}{|c|c|c|c|}
\hline Characteristic & Control $(\mathrm{n}=33)$ & $\operatorname{Yoga}(\mathrm{n}=33)$ & $\mathrm{p}$ \\
\hline Age & $51.51 \pm 8.15$ & $53.15 \pm 11.59$ & 0.5098 \\
\hline Men & 31 & 26 & - \\
\hline Women & 2 & 7 & - \\
\hline Total cholesterol (mmol/L) & $166.53 \pm 58.22$ & $165.83 \pm 44.05$ & 0.9615 \\
\hline Total cholesterol $>240 \mathrm{mmol} / \mathrm{L}$ & 4 & 1 & - \\
\hline Triglycerides (mmol/L) & $147.15 \pm 63.20$ & $171.83 \pm 71.91$ & 0.2054 \\
\hline Triglycerides $>200 \mathrm{mmol} / \mathrm{L}$ & 6 & 7 & - \\
\hline HDL (mmol/L) & $34.03 \pm 9.98$ & $32.75 \pm 10.44$ & 0.6613 \\
\hline $\mathrm{HDL}<40 \mathrm{mmol} / \mathrm{L}$ & 21 & 16 & - \\
\hline LDL (mmol/L) & $106.46 \pm 53.02$ & $103.66 \pm 34.59$ & 0.8251 \\
\hline $\mathrm{LDL}>160 \mathrm{mmol} / \mathrm{L}$ & 4 & 0 & - \\
\hline RBS & $166.53 \pm 125.48$ & $168.29 \pm 93.16$ & 0.6202 \\
\hline Diabetics (RBS > 200 mg/dL) & 8 & 5 & - \\
\hline Current smokers & 14 & 17 & - \\
\hline NYHA class II & 18 & 14 & - \\
\hline NYHA class I & 15 & 19 & - \\
\hline
\end{tabular}

HDL, high-density lipoprotein; LDL, low-density lipoprotein; NYHA, New York Heart Association; RBS, random blood sugar. 


\section{Statistical analysis}

All data used in this article were tested for normality and were found that it was not normally distributed. Baseline comparison between control and yoga groups using the Wilcoxon-Rank sum test was performed to identify any baseline differences in the data. Data collection and analysis were performed using Statistical Package for the Social Sciences (SPSS) for Windows (version 17.0; SPSS, Chicago, IL). All the tests were two tailed, and the significance threshold was $p=0.05$.

\section{Ethical clearance and informed consent}

The study was cleared by the Institutional Ethics Committees of Swami Vivekananda Yoga Anusandhana Samsthana (SVYASA) University, Bangalore, as well as Sri Jayadeva Institute of Cardiovascular Sciences and Research, Bangalore, India. The trial was registered with the Central Trial Registry of India (CTRI/2015/02/005563). Written informed consent was obtained from all participants at the time of enrollment.

\section{Results}

A total of 867 subjects were screened; 233 met the selection criteria, of which 66 agreed to participate in the study (Supplementary Figure S1). Participants were 52.3 years old on average $(\mathrm{SD}=9.9)$ and predominantly male $(86.3 \%)$. These subjects were randomly allocated to control $(n=33)$ and yoga $(n=33)$ groups. Of these subjects, 32 were classified as class II and 34 as class I using NYHA criteria. There was no statistically significant difference across baseline parameters between the control and yoga group for LVEF, CDS, and HAM-A (Supplementary Table S2).

\section{No significant change in the cardiac function of the patients on yoga practice}

The difference in the LVEF levels between the two groups did not reach statistical significance $(U=420.500, p$ value $=0.218$ ). However, when the authors conducted withingroup analysis, they observed a marginal improvement $(1.6 \%)$ in the mean LVEF levels in the yoga group (52.53 \pm 0.832$)$ compared to the control group (50.9 \pm 0.859$)$ (Fig. 3E).

\section{Significant reduction in CDS and HAM-A scores in the yoga practice group compared to control}

Cardiac patients assigned to the yoga group showed statistically significant reduction in $\operatorname{CDS}(U=71, p=0.00)$ and HAMA $(U=128, p=0.00)$ compared to control patients (Fig. 3A, B).
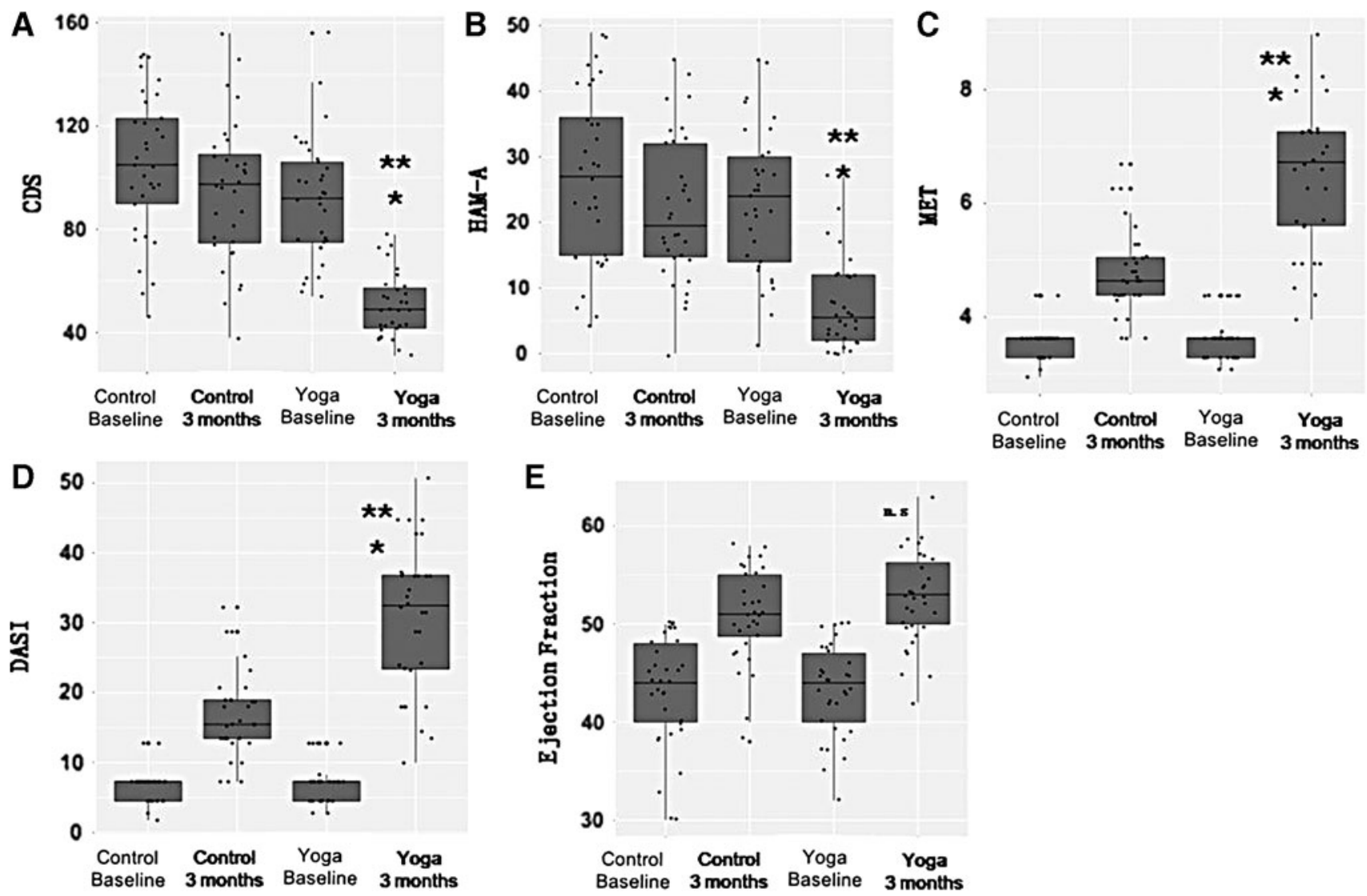

FIG. 3. Significant reduction in depression, anxiety, and increased QOL on yoga practice post MI. Box-plot illustrating significant reduction in (A) CDS and (B) HAM-A in yoga practicing group at 3 months compared to control. In addition, there is a significant increase in QOL parameters (C) MET and (D) DASI. There is a small increase in (E) LVEF in cardiac subjects in control and yoga practice 3 months follow-up but it did not achieve significance $(p=0.218)$. ${ }^{*} p$-value $<0.05$; $* * p$-value $<0.01$, *** $p$-value $<0.001$. Total samples $n=66$. CDS, Cardiac Depression Scale; DASI, Duke Activity Status Index; HAM-A, Hamilton Anxiety Rating Scale; LVEF, left ventricular ejection fraction; MET, metabolic equivalent; MI, myocardial infarction; QOL, quality of life. 

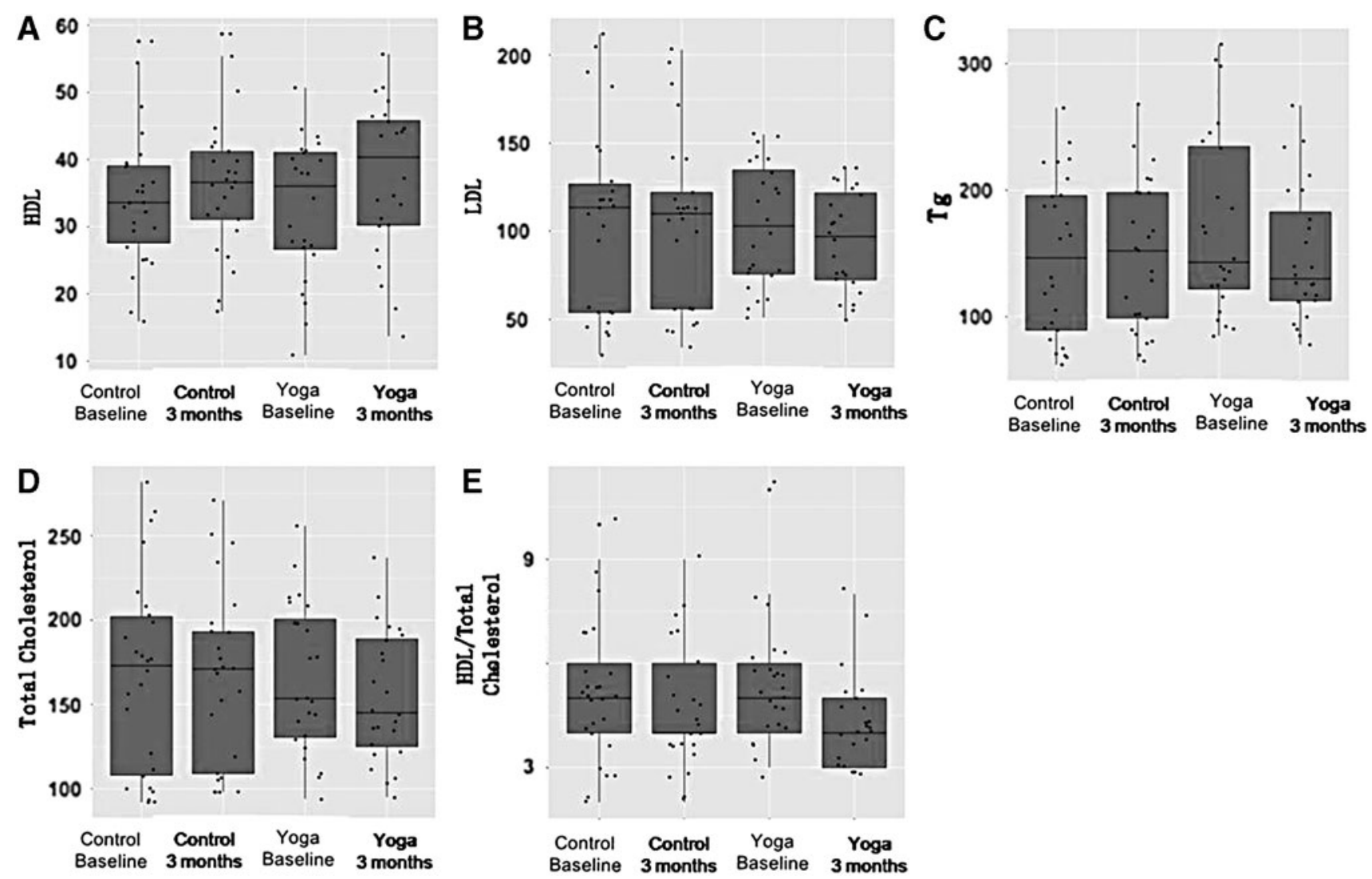

FIG. 4. No significant change in biochemical parameters on yoga practice post MI. Box-plot illustrating (A) HDL, (B) LDL, (C) Tg, (D) total cholesterol, and (E) HDL/total cholesterol ratio across control band yoga at baseline and after 3 months follow-up. There was no significant change in these biochemical parameters on yoga intervention post MI. Total samples $n=66$. HDL, high-density lipoprotein; LDL, low-density lipoprotein; MI, myocardial infarction.

\section{Significant increase in QOL of cardiac patients on yoga practice}

DASI estimates functional capacity in patients with heart failure, and a series of points in the DASI values were used to determine cardiac functional capacity based on METs. DASI and MET scores were determined for cardiac patients undergoing yoga practice and control. The authors observed a significant increase in the DASI $(U=146, p=0)$ and MET $(U=136, p=0)$ score when cardiac patients practiced yoga along with their medications compared to controls, who were on medications only (Fig. 3C, D).

\section{No significant change in biochemical outcome of cardiac patients on yoga practice}

The different biochemical lipid parameters were measured at baseline and 3 months in control and yoga groups. The authors observed a reduction in LDL, triglycerides, total cholesterol, and HDL/total cholesterol ratio and increase in HDL in 3 months yoga practice group compared to control (Fig. 4). Although not statistically significant, there was a greater improvement in the lipid profile of patients on yoga practice compared to control.

\section{Discussion}

Cardiac function was the primary outcome of this study, and LVEF was taken as a measure of cardiac function. A nonsig- nificant improvement of LVEF in the yoga practitioners following 12 weeks of supervised practice compared to control group participants was observed. Their results are in contrast with that of Sadeghi et al., whose work on cardiac rehabilitation for left ventricular dysfunction patients noticed a significant rise in the LVEF following 8 weeks of exercise training involving treadmill and ergometer. ${ }^{45}$ The authors speculate that this contrast in results in analyzing LVEF activity could be due to more intense activity in the Sadeghi et al. study while their protocol was less intense. Studies have documented improved left ventricular dysfunction when yoga therapy was coupled with standard medical therapy in patients with cardiac failure. ${ }^{46}$

Joshi et al. investigated the effect of improved LVEF on the long-term prognosis of patients with ischemic left ventricular systolic dysfunction, where they have indicated that a threshold of $8 \%$ improvement in the LVEF could be considered significant for the patient survival and heart failure hospitalization even though the clinical meaningful improvement of LVEF is considered to be higher than $5 \%$ in most of the studies. ${ }^{46,47}$ The reasons for lack of statistical significance in LVEF, as documented in previous studies, include inconsistent timing of repeat LVEF assessment, time of symptom onset to a successful intervention, anterior location of the infarct, female sex, and underestimation by the echo technician, especially in large volume chambers. 7,48

The study also investigates for the first time the detailed usage of CDS, HAM-A, and DASI questionnaires in Indian 
cardiac patients. This study is exploring the feasibility of introducing yoga as a component of cardiac rehabilitation in India. The authors show that the cardiac rehabilitation program, implemented through "yoga practice" showed a significant reduction in CDS and HAM-A scores and an increase in QOL through the DASI and MET scores in Indian cardiac patients. The concept of cardiac rehabilitation is less known in the eastern part of the world, including India. Furthermore, in places where it is offered, the patient referral and participation are not encouraging. Yoga practice is culturally better accepted in India, and patients adhere to the protocol well. ${ }^{49} \mathrm{~A}$ study on IAYT practice in post CABG patients showed a significant improvement in LVEF after 6 months and 1 year after surgery. ${ }^{8}$ In their study, LVEF outcomes were not similar to those in the Raghuram et al. study. There was a very slight improvement $(1.6 \%)$ in the LVEF but did not reach statistical significance compared with the control. However, it would be interesting to know and investigate if the $1.6 \%$ change is indicative of a clinically important change to acute MI patients.

Research findings suggest that both physiologic and psychologic components have to be focused simultaneously, and modification of one risk factor may not translate automatically to benefit the other. ${ }^{50}$ Rehabilitation program in cardiac conditions attempts to improve (1) depression and anxiety, (2) QOL and exercise capacity, and (3) cardiac function using yoga practice integrated into lifestyle therapy. These parameters can be assessed by different questions and their scoring patterns.

\section{Depression and anxiety}

The effect of IAYT on the psychologic status of their patients as assessed by CDS and HAM-A was improved substantiating studies that demonstrated a $40 \%-70 \%$ reduction in depression, anxiety, and hostility following cardiac rehabilitation. ${ }^{18}$ Yoga is known for its positive psychologic effects, and as its definition states "Yoga is the suppression of modification of mind". Patients perceived that practicing yoga and meditation helps calm thoughts, emotions, and ease physical discomforts, supporting its emerging beneficial role in health issues like hypertension and coronary heart disease (CHD). ${ }^{27,51,52}$

\section{QOL and exercise capacity}

Their study population exhibited a statistically significant improvement in the DASI and its derivative, MET scores, following 12 weeks of supervised IAYT compared to the control group. This is consistent with the results where homebased rehabilitation was tried in CHD patients, in which there was a significant improvement in the participants of the rehabilitation group, even though the quantum of improvement was comparatively low in the aged population (between 66 and 75 years of age). ${ }^{53}$ Energy expenditure has been estimated for various activities of daily living and alternative forms of physical activities. Tai Chi Chuan, one of the Chinese martial arts, demand a MET level of around 4.6, which is equivalent to walking on a flat surface at a speed of 3.7 miles per hour. Yoga, which shares many similarities with Tai Chi, expenses up to $3.3 \mathrm{MET}$ for an entire session of $1 \mathrm{~h}$ while achieving $50 \%-77 \%$ of maximum predicted heart rate. This lower level of physical activity would be a better alternative for the sedentary individuals and patient population. ${ }^{54}$
Yoga-based rehabilitation is low impact, does not demand any special types of equipment, requires less space for its practice compared to the conventional aerobic exercises, and has limited harmful side effects and, hence, may be a better alternative to conventional exercises for patients considered for cardiac rehabilitation. Yoga emphasizes more on relaxation, meditation, and awareness, which make it a pleasurable exercise experience rather than strenuous and may be encouraging to the patient population for long-term adherence to practice. ${ }^{33,54}$ There was a good improvement in bodily pain, general health perception, physical and social functioning, and overall on the health-related QOL in the Chinese participants with metabolic syndrome who completed a 12-week yoga training session. ${ }^{33}$

The authors speculate that the supervised yoga training by the health care professionals could have helped the patients of the yoga group overcome apprehension. This, in turn, would have motivated the patients to attempt activities, which they would not have done otherwise, even though their health status would permit. This could be one of the reasons for the statistically significant difference in the QOL outcome between the groups in their study.

Even though the lipid profile of patients who practiced IAYT was improved compared to the control group, the values did not reach statistical significance. Their results are consistent with the previously published study exploring the effects of yogabased cardiac rehabilitation in post CABG patients. ${ }^{8}$ Exercises lead to an increase in the levels of HDL cholesterol and thereby preventing further atherosclerotic changes. ${ }^{52}$ The low levels of physical activity achieved with the practice of yoga are suitable to achieve metabolic fitness with an improvement in indices such as lipid profile and insulin resistance. ${ }^{54}$

Lau et al. who used 60-min yoga sessions over 12 weeks indicated that longer training durations would help to increase the levels of serum HDL especially in patients with higher baseline HDL levels. ${ }^{33}$ Yoga practice improved metabolic parameters, including serum triglycerides in Chinese adults. ${ }^{33}$ However, in the Multicenter Lifestyle Demonstration Project (MLDP) focusing on exercise and stress management, conducted in the United States, the HDL cholesterol and triglycerides did not show much improvement from the baseline for 1 year. ${ }^{55}$

In another study involving the Multisite Cardiac Lifestyle Intervention Program (MCLIP), 12 weeks of lifestyle modification and stress management showed lower perceived stress and increased psychologic well-being with reduced dietary fat intake. This better stress management was related to reduced triglycerides, total cholesterol/HDL-C ratio, and Hemoglobin Alc in CHD patients with a history of diabetes. ${ }^{16}$ Bandura et al. suggested that this improvement in psychologic well-being may lead to improved eating behavior, which further contributes to improved biochemical profile. ${ }^{56}$

Previous studies have demonstrated inconsistent effects of exercise on LDL cholesterol and that following a couple of months of aerobic exercise, LDL cholesterol, in general, did not change significantly, but those subfractions of LDL which were atherogenic did. Lower baseline levels of triglycerides yielded fewer improvements following aerobic exercise training. Factors contributing to the response of triglycerides and HDL cholesterol to exercise training include body weight, body fat, cardiovascular fitness, training status, regional lipid concentration, dietary changes, and genetic factors. Blood 
sample collection time, blood test technology, as well as exercise time, could be additional contributing factors. ${ }^{31}$

\section{Mechanism}

Physical training improves fibrinolysis and myocardial perfusion, which in turn improves systolic function, as well as ejection fraction, as a consequence of an improvement in the myocardial strength. ${ }^{45}$ Studies proposed a mechanism on how yoga could reduce stress arousal. Chronic stress, psychologic distress, depression, and anxiety have a vital role in the pathogenesis of atherosclerosis.

Yoga leads to a reduction in the sympathetic activity, which explains the improvement in anxiety, depression, perceived stress, and negative effect. Correction in mindset by regular introspection at physical, mental, emotional, and intellectual levels is achieved through autonomic regulation with yoga practice. Yoga helps modulation of neuro-endocrineimmunologic pathways and affects the hypothalamo-pituitaryadrenal axis. ${ }^{8}$ Physical postures of yoga, combined with meditation and breathing exercises, decrease the sympathetic activity in turn leading to a reduction in the ventricular filling pressure. ${ }^{27}$

The proposed mechanism may help reduce the load on the myocardium. In addition to the predominance of the parasympathetic state, yoga may also promote the effective extraction of oxygen by peripheral tissues. When a muscle is stretched, the oxygen consumption increases. ${ }^{57}$ The 8-week health effects of yoga training showed increased muscle strength (31\%), muscular endurance (57\%), flexibility (88\%), oxygen uptake (7\%), and reduced cardiovascular risk in healthy adults. ${ }^{35}$

The strengths of the current study lie in the fact that this is the preliminary RCT assessing the effectiveness of IAYT in post-MI patients with left ventricular dysfunction in India. This study uses CDS, HAM-A, and DASI questionnaires in Indian cardiac patients. Supervised yoga intervention, high patient retention, and blinded raters for LVEF are additional strengths worth mentioning (Supplementary Table S3). Their study had several limitations such as a small sample, gender-biased to males, absence of information on confounding factors such as diet, absence of an active control group, and an absence of a plan to compare with any other form of exercise. There was no method used to assess the adherence of either the patients or caregivers to the intervention. In addition, the contribution of medication in the improvement of LVEF was also not documented. The low physical demand of the intervention, estimated at around 3.3 METs, not sufficient enough to effectively target the cardiovascular system may not be ruled out.

There are no adverse events documented with the practice of IAYT protocol under consideration in post-MI patients with NYHA class I and II left ventricular dysfunction. Future studies may be designed with larger sample size, equal male to female participants of a conventional cardiac rehabilitation program as a control group, more sophisticated methods of LVEF measurement, including stress echocardiogram, assessment of the extent of the viability of the myocardium through myocardial perfusion studies/thallium scans, and inclusion of a longer duration of the supervised IAYT practice.

\section{Conclusion}

This study indicated that integration of yoga practice in cardiac rehabilitation program is feasible. Yoga therapy used as an adjuvant to conventional pharmacologic management has no additional benefits in improving cardiac function (LVEF) compared to standard care. However, it may assist in reducing depression and anxiety, as well as improve overall QOL, in post MI patients. The study also establishes the utility of CDS, HAM-A, and DASI questionnaires on Indian cardiac patients. Future studies with larger sample sizes utilizing more diverse population and longer duration of yoga practice are recommended.

\section{Acknowledgments}

The authors thank Dr. C.N. Manjunath, Director of Sri Jayadeva Institute of Cardiovascular Sciences and Research, Bengaluru and Dr. H.R. Nagendra, Chancellor of Swami Vivekananda Yoga Anusandhana Samsthana (S-VYASA) for providing them the opportunity to conduct this study. They also thank all patients and treating team, for their individual and collective contribution in completing this study.

\section{Human Subjects/Informed Consent Statement}

All procedures followed were in accordance with the ethical standards of the responsible committee on human experimentation. Informed consent was obtained from all patients for being included in the study.

\section{Author Disclosure Statement}

No competing financial interests exist.

\section{Funding Information}

No funding was received for this article.

\section{Supplementary Material}

Supplementary Figure S1

Supplementary Table S1

Supplementary Table S2

Supplementary Table S3

\section{References}

1. Yusuf S, Reddy S, Ônpuu S, et al. Global burden of cardiovascular diseases: Part II: Variations in cardiovascular disease by specific ethnic groups and geographic regions and prevention strategies. Circulation 2001;104: 2855-2864.

2. Prabhakaran D, Jeemon P, Roy A. Cardiovascular diseases in India: Current epidemiology and future directions. Circulation 2016;133:1605-1620.

3. Fernandez C, Sandin M, Sampaio JL, et al. Plasma lipid composition and risk of developing cardiovascular disease. PLoS One 2013;8:e71846.

4. Hippisley-cox J, Coupland C. Diabetes treatments and risk of heart failure, cardiovascular disease, and all cause mortality: Cohort study in primary care BMJ 2016;354:i3477.

5. Chauvet-Gelinier J-C, Bonin B. Stress, anxiety and depression in heart disease patients: A major challenge for cardiac rehabilitation. Ann Phys Rehabil Med 2017;60: 6-12.

6. Tanaka S, Hayashi T, Kihara Y, et al. Standard measurement of cardiac function indexes. J Med Ultrason 2006;33: 123-127. 
7. Gibson DG. Study of left ventricular function by echocardiography. Ann Acad Med Singapore 1981;10:110-114.

8. Raghuram N, Parachuri VR, Swarnagowri MV, et al. Yoga based cardiac rehabilitation after coronary artery bypass surgery: One-year results on LVEF, lipid profile and psychological states - A randomized controlled study. Indian Heart J 2014;66:490-502.

9. El-Yassin HD, Hasso NMA, Al-Rubayi HA. Lipid profile and lipid peroxidation pattern pre and post exercise in coronary artery disease. Turkish J Med Sci 2005;35:223-228.

10. DeFrances CJ, Lucas CA, Buie VC, et al. 2006 national hospital discharge survey. Natl Health Stat Rep 2008;5: $1-20$.

11. Meikle PJ, Wong G, Barlow CK, et al. Plasma lipid profiling shows similar associations with prediabetes and type 2 diabetes. PLoS One 2013;8:e74341.

12. Gaziano TA, Bitton A, Anand S, et al. Growing epidemic of coronary heart disease in low- and middle-income countries. Curr Probl Cardiol 2010;35:72-115.

13. Coelho VG, Caetano LF, Liberatore Júnior RDR, et al. Lipid profile and risk factors for cardiovascular diseases in medicine students. Arq Bras Cardiol 2005;85:57-62.

14. Haque A, Yusoff F, Ariffin M, et al. Lipid Profile of the coronary heart disease (CHD) patients admitted in a hospital in Malaysia. J Appl Pharm Sci 2016;6:137-142.

15. Dasgupta J, Dasgupta S, Gayen R, et al. Study of lipid profile in patients of coronary artery disease among rural population. IOSR J Pharm Biol Sci 2015;10:51-54.

16. Deubenmier JJ, Mendell N, Merritt-Wonden T, et al. The contribution of changes in diet, exercise and stress management to changes in coronary risk in women and men in Multisite Cardiac Lifestyle Intervention program. Ann Behav Med 2007;33:57-68.

17. Ren Y, Jia J, Sa J, et al. Association between N-terminal pro B-type natriuretic peptide and depressive symptoms in patients with acute myocardial infarction. Chin Med J (Engl) 2017;130:542.

18. Contractor AS. Cardiac rehabilitation after myocardial infarction. J Assoc Physicians India 2011;59(Suppl.):51-55.

19. Nielsen TJ, Vestergaard M, Christensen B, et al. Mental health status and risk of new cardiovascular events or death in patients with myocardial infarction: A population-based cohort study. BMJ Open 2013;3:e003045.

20. Rozanski A, Blumenthal JA, Davidson KW, et al. The epidemiology, pathophysiology, and management of psychosocial risk factors in cardiac practice: The emerging field of behavioral cardiology. J Am Coll Cardiol 2005;45:637-651.

21. Feng H-P, Chien W-C, Cheng W-T, et al. Risk of anxiety and depressive disorders in patients with myocardial infarction. Medicine (Baltimore) 2016;95:34.

22. Ontario HQ. Screening and management of depression for adults with chronic diseases: An evidence-based analysis. Ont Health Technol Assess Ser 2013;13:1-45.

23. Evangelista LS, Lee J, Moore AA, et al. Examining the effects of remote monitoring systems on activation, selfcare, and quality of life in older patients with chronic heart failure. J Cardiovasc Nurs 2015;30:51-57.

24. Saengsiri A, Hacker ED. Conducting quality of life research in people with coronary artery disease in non- Englishspeaking countries: Conceptual and Operationalization Issues. J Cardiovasc Nurs 2015;30:74-84.

25. Dobbels F. Does every cardiologist need a psychologist? Eur Heart J 2007;28:2964-2966.
26. Corrà U, Piepoli MF, Carré F, et al. Secondary prevention through cardiac rehabilitation: Physical activity counselling and exercise training. Key components of the position paper from the Cardiac Rehabilitation Section of the European Association of Cardiovascular Prevention and Rehabilitat. Eur Heart J 2010;31:1967-1974.

27. Pullen PR. The Benefits of Yoga Therapy for Heart Failure Patients (Dissertation). Atlanta (GA): Georgia State University, 2009.

28. Lennie TA, Moser DK, Biddle MJ, et al. Nutrition intervention to decrease symptoms in patients with advanced heart failure. Res Nurs Health 2013;36:120-145.

29. Kondredddy R, Chenak A, Akula US, et al. Study of lipid profile in coronary heart disease patients in Libya. J Basic Med Allied Sci 2012;1:1-9.

30. Ferduos BA, Sultana N, Ahmed S, et al. Serum lipid profile in ischemic heart disease: A cross-sectional analytical study. Bangladesh J Med Biochem 2014;7:14-16.

31. Wang Y, Xu D. Effects of aerobic exercise on lipids and lipoproteins. Lipids Health Dis 2017;16:132.

32. Innes KE, Vincent HK, Taylor AG, et al. Chronic stress and insulin resistance- related indices of cardiovascular disease risk, part 2: A potential role for mind-body therapies. Altern Ther Health Med 2007;13:44-51.

33. Lau C, Yu R, Woo J. Effects of a 12-week Hatha yoga intervention on metabolic risk and quality of life in Hong Kong Chinese adults with and without metabolic syndrome. PLoS One 2015;10:1-18.

34. Faul F, Erdfelder E, Lang A-G, et al. G*Power 3: A flexible statistical power analysis program for the social, behavioral, and biomedical sciences. Behav Res Methods 2007; 39:175-191.

35. Hari Krishna B, Pal P, Pal GK, et al. A randomized controlled trial to study the effect of Yoga therapy on cardiac function and $\mathrm{N}$ Terminal Pro BNP in heart failure. Integr Med Insights 2014;9:1-6.

36. Saghaei M. An overview of randomization and minimization programs for randomized clinical trials. J Med Signals Sens 2011;1:55-61.

37. Sharma S, Pailoor S, Choudhary Ram N, et al. Development of a yoga module targeting cardiovascular health for patients with post-myocardial left ventricular dysfunction in India. Complement Ther Med 2019;42: 170-177.

38. Amiel J-B, Grümann A, Lhéritier G, et al. Assessment of left ventricular ejection fraction using an ultrasonic stethoscope in critically ill patients. Crit Care 2012;16: R29.

39. Storti FC, Moffa PJ, Uchida AH, et al. New prognostic score for stable coronary disease evaluation. Arq Bras Cardiol 2011;96:411-419.

40. Vibulchai N, Thanasilp S, Preechawong S, et al. Validation of the Thai version of the Duke Activity Status Index in patients with a previous myocardial infarction. Asian Biomed 2014;8:623-629.

41. George MJ, Kasbekar SA, Bhagawati D, et al. The value of the Duke Activity Status Index (DASI) in predicting ischaemia in myocardial perfusion scintigraphy-A prospective study. Nucl Med Rev 2010;13:59-63.

42. Hamilton $\mathrm{M}$. The assessment of anxiety states by rating. $\mathrm{Br}$ J Med Psychol 1959;32:50-55.

43. Ski CF, Thompson DR, Hare DL, et al. Cardiac Depression Scale: Mokken scaling in heart failure patients. Health Qual Life Outcomes 2012, 10:141:1-5. 
44. Hare DL, Davis CR. Cardiac Depression Scale: Validation of a new depression scale for cardiac patients. J Psychosom Res 1996;40:379-386.

45. Sadeghi M, Garakyaraghi M, Khosravi M, et al. The impacts of cardiac rehabilitation program on echocardiographic parameters in coronary artery disease patients with left ventricular dysfunction. Cardiol Res Pract 2013;2013:201713.

46. Joshi K, Alam I, Ruden E, et al. Effect of improvement in left ventricular ejection fraction on long-term survival in revascularized patients with ischaemic left ventricular systolic dysfunction. Eur J Echocardiogr 2011;12:454-460.

47. Breathett K, Allen LA, Udelson J, et al. Changes in left ventricular ejection fraction predict survival and hospitalization in heart failure with reduced ejection fraction. Circ Heart Fail 2016;9:e002962.

48. Heikki H, Raatikainen P, Sinnecker D, et al. Change in Left Ventricular Ejection Fraction following first myocardial infarction and outcome. JACC Clin Electrophysiol 2018;4: 672-682.

49. Tiwari S, Pandey N. The Indian concepts of lifestyle and mental health in old age. Indian J Psychiatry 2013;55:288.

50. Suchday S, Dziok M, Katzenstein M, et al. The effects of meditation and yoga on cardiovascular disease. In: Dornelas EA, eds. Stress Proof the Heart. New York, NY: Springer, 2012.

51. Hartley L, Dyakova M, Holmes J, et al. Yoga for the primary prevention of cardiovascular disease (Review). Cochrane Database Syst Rev 2014:CD010072.

52. Longfellow W. Standard and alternative adjunctive treatments in cardiac rehabilitation. Card Rehabil Adjunctive Treat 1993;20:198-212.
53. Oerkild B, Frederiksen M, Hansen JF, et al. Home-based cardiac rehabilitation is an attractive alternative to no cardiac rehabilitation for elderly patients with coronary heart disease: Results from a randomised clinical trial. BMJ Open 2012;2:e001820.

54. Hagins M, Moore W, Rundle A. Does practicing Hatha yoga satisfy recommendations for intensity of physical activity which improves and maintains health and cardiovascular fitness? BMC Complement Altern Med 2007;7:40.

55. Koertge J, Weidner G, Elliott-Eller M, et al. Improvement in medical risk factors and quality of life in women and men with coronary artery disease in the Multicenter Lifestyle Demonstration Project. Am J Cardiol 2003;91:13161322.

56. Pischke CR, Scherwitz L, Weidner G, et al. Long-term effects of lifestyle changes on well-being and cardiac variables among coronary heart disease patients. Health Psychol 2008;27:584-592.

57. Tran MD, Holly RG, Lashbrook J, et al. Effects of Hatha yoga practice on the health-related aspects of physical fitness. Prev Cardiol 2001;4:165-170.

Address correspondence to:
K.N. Srihari Sharma, MPT
College of Physiotherapy
School of Health Sciences
Dayananda Sagar University Kumaraswamy Layout
Bangalore 560111
India
E-mail: srihari-physio@dsu.edu.in

Address correspondence to:

College of Physiotherapy

School of Health Sciences

Bangalore 560111

India

E-mail: srihari-physio@dsu.edu.in 